\title{
Performance evaluation of some Hybrid ARQ schemes in IEEE 802.11a Networks
}

\author{
Emilio Calvanese Strinati ${ }^{1,2}$, Sébastien Simoens ${ }^{1}$ and Joseph Boutros ${ }^{2}$ \\ strinati, simoens@crm.mot. com; boutrosdenst. fr \\ ${ }^{1}$ Motorola Labs Paris, Saint-Aubin 91193 Gif-sur-Yvette France - tel: +33 (0)1 69352561 \\ ${ }^{2}$ Ecole Nationale Supérieure des Télécommunications 75634 Paris France - tel: +33 (0)1 45817678
}

\begin{abstract}
This paper investigates how a type II Hybrid ARQ (HARQ) scheme combined with Adaptive Modulation and Coding $(A M C)$ can improve the throughput on top of the MAC layer, by comparing it to the existing type I HARQ + AMC mechanism in the IEEE 802.11 a context. The studied type II HARQ+AMC strategy relies on Incremental Redundancy (IR) by use of Rate Compatible Punctured Codes as well as Chase Combining. The performance of the various strategies is mainly affected by two parameters. One of these is the inaccuracy of the link quality predictor, since the SNIR inherently fluctuates due to fading and interference; the other is the MAC overhead, which is especially important in IEEE 802.11 a due to the CSMA/CA protocol. From the simulation results presented in this paper, it can be observed that even when MAC overhead is taken into account, the type II HARQ+AMC strategy still provides up to $1.5 \mathrm{~dB}$ gain with respect to current type I HARQ + AMC scheme.
\end{abstract}

\section{Introduction}

In wireless communications, adaptive mechanisms are needed to adjust the transmission parameters such as the constellation size and the coding rate to the momentary link quality which fluctuates due to fading and interference. In current OFDM Wireless LAN standards such as IEEE 802.11a [1] and ETSI BRAN HIPERLAN/2 [4], an Adaptive Modulation and Coding (AMC) [7] scheme enables the bit rate of the Physical Layer to range from 6 to $54 \mathrm{Mbit} / \mathrm{s}$. In addition, a Hybrid ARQ [10] of type I (HARQ-I) is specified, which consists in retransmitting all the convolutionally coded bits corresponding to a packet as long as the latter is detected as erroneous by the receiver. IEEE 802.11a and HIPERLAN/2 ARQ strategies are different; while the first uses Stop and Wait and the second Selective Repeat, their parameters can be designed to approach the ideal ARQ performance [13]. The goal of AMC is to select the constellation size and coding rate in order to maximize the throughput given the link state under some constraints such as Qual- ity of Service. Thus, AMC requires a link quality metric. In [12], we computed the throughput of an isolated HIPERLAN/2 connection when using the instantaneous SNR as the link quality metric, assuming ideal ARQ and neglecting the overhead of the Medium Access Control (MAC) layer. In [6], a combination of Hybrid ARQ of type II with AMC is studied with a view to further increase the throughput. This HARQ-II scheme relies on Incremental Redundancy (IR) which was introduced by Mandelbaum in [11], and makes use of Rate Compatible Punctured Codes (RCPC) as described in [8]. In [6], the authors neglect the MAC overhead and propose a blind scheme which starts transmitting at the highest coding rate and constellation size, removing the need for a link quality metric. Actually, the 802.11 MAC layer introduces a significant overhead at each transmission, which has a large impact on the 802.11a throughput. This must be taken into account in the design of a combination of IR and AMC.

In this paper we propose a type II HARQ scheme combined with AMC which takes into account 802.11a constraints. Our scheme relies on both IR and Chase Combining [3]. The decision on the initial constellation and coding rate is based on Signal to Noise plus Interference Ratio (SNIR) as link quality metric. As a result, the average number of retransmissions is reduced and the MAC throughput is improved. The effect of interference and SNIR prediction errors are evaluated by simulation. Since the puncturing matrices defined by the 802.11a standard do not respect the rate compatibility restriction, we introduce a new puncturing pattern for the code rate $\frac{2}{3}$. This pattern is compatible with rate $\frac{3}{4}$ and also with rate $\frac{9}{16}$ of HIPERLAN/2, so that IR would be feasible with both physical layer specifications, at the expense of a slight degradation of the BER performance

The paper is organized as follows. In Section II an overview of the characteristics of the studied Hybrid ARQ schemes is given. At the end of the section the proposed type II HARQ combined with AMC is described. Section III analyzes the limitation due to the IEEE 802.11 a standard to the rate compatibility restriction and describes the new puncturing pattern to solve this problem. Assumptions of 
the performance study and simulation results are presented in Section IV. Finally, conclusions are drawn in section V.

\section{Hybrid ARQ Schemes}

The two following retransmission strategies were compared:

1. A type I HARQ combined with AMC mechanism, compatible with the IEEE 802.11a standard [1]. In the following it will be called: HARQ-I+AMC.

The receiver discards the packet upon erroneous reception (i.e. when errors remain after Viterbi decoding) and it asks for an entirely new retransmission. There is no combining of earlier and later versions of the packet, each one is standalone. Retransmission takes place at either the same or lower code rate until the packet is correctly received or a pre-set number of retransmissions have taken place or until a delay constraint has been exceeded. This method does not require a buffering of corrupted packets at the receiver for later decoding since only the correctly received data that has not already been sent to the upper layers is stored in memory. Therefore this scheme is relatively simple to implement. The disadvantage of type I HARQ scheme is that once the coding rate is chosen, all the parity bits for error correction are transmitted even if they are not all needed, therefore the channel use efficiency is reduced. We assume in this reference scheme that the AMC is only based on SNIR estimate. Consequently the constellation and coding rate remain fixed during all successive retransmissions of the packet, provided the SNIR remains constant. Note that we implicitely assume that there exists a means to signal the SNIR, although this feature is not specified in the standard.

2. A type II HARQ which relies on IR combined with AMC mechanism. In the following it will be called HARQ-IR+AMC. This HARQ scheme gains from both the advantages of RCPC codes [8] and Chase combining [3]. The operation of an IR strategy with RCPC is the following: the codeword $C_{0}$ obtained by the convolutional encoding of the packet is punctured in order to obtain a set of codewords $C_{i}$ corresponding to different coding rates $R_{i}>R_{0}$. The codes obtained are RCPC, meaning that the puncturing matrices are chosen such that the coded bits of $C_{i}$ are included in $C_{j}$ if $R_{i}>R_{j}$. An IR strategy based on RCPC first transmits at the highest code rate $R_{I}$. If the transmission fails, the next lower code rate $R_{I-1}$ is obtained by sending only the coded bits which differ between $C_{I}$ and $C_{I-1}$. With each retransmission, more and more punctured bits are transmitted. Retransmissions will continue until all the coded bits of codeword $C_{0}$ are received. The procedure can be restarted if $C_{0}$ cannot be decoded. At any moment, it can be interrupted in case of successful decoding or if a delay constraint is exceeded. To summarize, IR allows the effective code rate to be gradually lowered until the packet can be successfully decoded.

The principle of the HARQ-II+AMC [5] is to start at an initial constellation and coding rate and to use a more robust mode at each retransmission either by reducing the coding rate (e.g. from $\frac{3}{4}$ to $\frac{2}{3}$ ) or by selecting a more robust constellation (e.g. from 64-QAM to 16QAM) when all the coded bits of $C_{0}$ have been received using the current constellation.

In the second strategy, the receiver performs the demapping of the bit-interleaved coded modulation to obtain bit metrics at each transmission attempt. After deinterleaving, these bit metrics have to be combined with those obtained from previous attempts, prior to Viterbi decoding. This combination requires careful attention. In [2], the noise variance $\sigma_{N}^{2}$ is simplified in the expression of the bit metrics of a convolutionally coded OFDM system. Here since the interference power $\sigma_{I}^{2}$ affecting each transmission can vary, the bit metrics must be weighted by the inverse of the momentary noise plus interference power $\frac{1}{\sigma_{I}^{2}+\sigma_{N}^{2}}$. The operation of the receiver is the following:

1. Bit metrics are initialized to the null value

2. If code rate equals $\frac{3}{4}$, then try to decode without combination. If decoding fails go to step 3 .

3. Combine with previously received bit metrics. Combination can be performed simply by adding the properly weighted metrics. If this is not the first attempt, go to step 4.

4. Try to decode the combined metrics. If it fails, send a negative acknowledgement to the sender.

The bit metrics combination can be viewed as a generalization of Chase combining [3], since Chase only addressed the soft combination of packets transmitted in the same mode. Also note that packets heavily corrupted by interference should not disturb the decoding since their relative weight in the combination is reduced. The effect of interference and SNIR estimation errors is addressed in section IV.2. Another issue is the MAC overhead due to the CSMA/CA access of 802.11. In the HARQ-IR+AMC studied in this paper, while the initial constellation is suggested by an AMC algorithm based on the knowledge of SNIR [12], the coding rate is always set to the highest allowed one: $R=\frac{3}{4}$. Justification for this choice is given in section IV.2. If the MAC overhead was negligible, the best strategy would always be 
to perform three steps of IR in order to reach successively $R=\frac{2}{3}, R=\frac{9}{16}$ and $R=\frac{1}{2}$ before changing of constellation. But the 802.11 MAC overhead makes such a strategy inefficient. Therefore, in the following, only rates $R=\frac{3}{4}$ and $R=\frac{1}{2}$ are kept. Although having a large number of rate compatible codes may not be a good strategy in the 802.11 context, it is interesting to see how a reasonable set of RCPC could be obtained by slightly modifying the $802.11 \mathrm{a}$ and HIPERLAN/2 standards. This is the purpose of the next section.

\section{Rate compatibility restrictions}

The IEEE 802.11a standard defines two puncturing patterns allowing coding rates $\frac{3}{4}$ and $\frac{2}{3}$ from the mother code rate $\frac{1}{2}$. These puncturing patterns are defined by the following puncturing matrices:

$$
\begin{gathered}
\mathbf{M}_{\frac{3}{4}}=\left[\begin{array}{llll}
1 & 1 & 0 \\
1 & 0 & 1
\end{array}\right] \\
\mathbf{M}_{\frac{2}{3}}=\left[\begin{array}{llllll}
1 & 1 & 1 & 1 & 1 & 1 \\
1 & 0 & 1 & 0 & 1 & 0
\end{array}\right]
\end{gathered}
$$

To perform any IR algorithm which uses the RCPC codes (Rate Compatible Punctured Convolutional codes), it is necessary to add a rate compatibility restriction on the puncturing rule. The rate compatibility criterion requires that the puncturing matrices be well chosen to make the coded bits of high rate punctured code belong to the lower rate code. An evaluation of the performance of the RCPC codes is given in [8].

The puncturing matrices defined by the IEEE 802.11a standard do not respect the rate compatibility restriction on the puncturing rule: where a zero is at the fourth position of the second row of $M_{\frac{2}{3}}$, a 1 is at the same position of $M_{\frac{3}{4}}$. With the interest in adopting an IR strategy that uses the RCPC codes, we investigated other puncturing matrices for the code rate $\frac{2}{3}$.

In particular, we considered a puncturing matrix for the code rate $\frac{2}{3}$ which respects the restrictions for the code rates of $\frac{3}{4}, \frac{2}{3}$ and $\frac{9}{16}$ (defined by both ETSI HIPERLAN/2 and IEEE 802.11a standards [1],[4]) with the aim of maintaining the physical layer compatibility between the two standards. It has to be noted that the performance of such puncturing matrices are not worse than that of a puncturing matrix not compatible with rate $\frac{9}{16}$. The puncturing matrix proposed for the rate $\frac{2}{3}$ is the following:

$$
\widetilde{\mathbf{M}_{\frac{2}{3}}}=\left[\begin{array}{llllllllllllllllll}
1 & 1 & 0 & 1 & 1 & 0 & 1 & 1 & 0 & 1 & 1 & 0 & 1 & 1 & 0 & 1 & 1 & 0 \\
1 & 1 & 1 & 1 & 0 & 1 & 1 & 0 & 1 & 1 & 1 & 1 & 1 & 0 & 1 & 1 & 1 & 1
\end{array}\right]
$$

We verified that the resulting convolutional code is not catastrophic. As it can be seen on figure 1 the performance loss due to the proposed puncturing pattern is less than 0.5 $\mathrm{dB}$ at every SNR. To conclude, the BER performance of the two puncturing matrices ( $M_{\frac{2}{3}}$ and $\widetilde{M_{2}}$ ) is almost the same, but with the remarkable difference that $\widetilde{M_{2}}$ respects the restriction on the puncturing rule to generate a RCPC code.

\section{Simulation results}

The performance in terms of average throughput versus average SNR was evaluated by simulation for both retransmission strategies: HARQ-I+AMC and proposed HARQIR+AMC. In order to enable a fair comparison, the same transmission modes are used. Table 1 provides the list of the modes. As explained in section II, rates $\frac{2}{3}$ and $\frac{9}{16}$ are not used. The following assumptions are made:

- the packet size is 1000 bytes

- the Cyclic Redundancy Check (CRC) enables perfect error detection and the feedback channel is error-free

- We neglected the additional signalling which would be required to implement HARQ-IR according to the standard

- The maximum number of transmission attempts is limited to 5

\begin{tabular}{|c|c|c|}
\hline Modulation & Code Rate & PHY bit rate \\
\hline \hline$B P S K$ & $1 / 2$ & $6 \mathrm{Mbit} / \mathrm{s}$ \\
\hline$B P S K$ & $3 / 4$ & $9 \mathrm{Mbit} / \mathrm{s}$ \\
\hline$Q P S K$ & $1 / 2$ & $12 \mathrm{Mbit} / \mathrm{s}$ \\
\hline$Q P S K$ & $3 / 4$ & $18 \mathrm{Mbit} / \mathrm{s}$ \\
\hline $16-Q A M$ & $1 / 2$ & $24 \mathrm{Mbit} / \mathrm{s}$ \\
\hline $16-Q A M$ & $3 / 4$ & $36 \mathrm{Mbit} / \mathrm{s}$ \\
\hline $64-Q A M$ & $1 / 2$ & $36 \mathrm{Mbit} / \mathrm{s}$ \\
\hline $64-Q A M$ & $3 / 4$ & $54 \mathrm{Mbit} / \mathrm{s}$ \\
\hline
\end{tabular}

Table 1: Simulated physical layer modes

Under these assumptions, simulations were performed in a typical indoor NLOS (Non Line Of Sight) channel with 50 ns RMS delay spread following the ETSI BRAN-A model and static terminals. The resulting average throughput versus SNR is plotted on figures 2- 5. As mentioned in section II, the initial constellation for HARQ-IR+AMC is selected according to the throughput maximization criterion of a HARQ-I+AMC scheme for the current SNR, but the starting coding rate is always $\frac{3}{4}$. The first motivation for this choice is that it enables to take advantage of IR, although the gain to be expected is reduced due to the MAC overhead. The gain should be higher when the predicted SNIR is lower than the actual one: in such cases the success 
probability of the first transmission is significant. Furthermore, the SNIR is not the best metric, since the Packet Error Rate (PER) depends not only on the SNIR, but also on the actual channel coefficients, as shown in [9]. If the PER performance of the current channel is better than average, once again the success probability of the transmission at rate $\frac{3}{4}$ can be high, obviously leading to a throughput gain. If the channel PER performance is worse than average, then a gain can also be expected: since our HARQ-IR strategy systematically reduces the mode at each attempt, a mode with low PER will be quickly reached, whereas the HARQ-I+AMC strategy will perform lots of retries in the inadequately selected initial mode.

\section{IV.1. HARQ-I+AMC versus HARQ-IR+AMC over a fading channel}

First, the comparison is performed without interference. A new independent channel impulse response is used at each new packet transmission, but it is assumed to remain constant during the successive retransmissions. This model is well adapted to the simulation of a static environment. The SNR fluctuates due to fading at each new transmission, but we assume that it can be perfectly predicted in order to perform AMC. The average PHY and MAC throughput are plotted respectively on fig. 2 and fig. 4 .

On figure 2, it can be observed that HARQ-IR+AMC outperforms HARQ-I+AMC by up to $1.5 \mathrm{~dB}$. For high order modulations (i.e. at high SNR) the gain is more important than for lower modulations (i.e. lower SNR). This is due to the fact that a larger number of IR steps are available in case of initial transmission failure. Unfortunately, the throughput gain on top of MAC layer is insignificant due to the MAC overhead, as shown on figure 4.

\section{IV.2. Impact of interference and SNIR prediction er- rors}

Contrary to fading which fluctuates slowly in WLAN, interference can produce large and quick changes of the SNIR. Besides, these variations are hardly predictable due to the random nature of traffic sources. In this section, we assume that the SNIR fluctuates randomly from one packet transmission or retransmission to the next. The SNIR fluctuation at each transmission attempt is modeled as a sequence of i.i.d. variables uniformly distributed between $-6 \mathrm{~dB}$ and $+6 \mathrm{~dB}$. We assume that the SNIR can be perfectly estimated in order to weight the soft bit metrics prior to combination with previous attempts. However, we add a random error to the SNIR prediction used by AMC. We take for the prediction error the same uniform distribution as the SNIR fluctuation. Therefore, the metrics weighting remains optimum, but the decision of the AMC on the initial transmission mode can be erroneous.
Our simulation results show how in presence of bad selection of the initial transmission mode due to a prediction error in the AMC, the HARQ-IR+AMC outperforms HARQI+AMC at both Physical and MAC layer. As shown respectively on figures 3 and 5 we observed up to $2.5 \mathrm{~dB}$ gain at Physical layer and still up to $1.5 \mathrm{~dB}$ gain at the MAC layer. These results validate the interest in adopting the proposed HARQ-IR+AMC strategy in a realistic transmission scenario.

\section{Conclusion and further work}

In this paper, we study an AMC scheme based on SNIR prediction combined with a type II Hybrid ARQ relying on IR adapted to the IEEE 802.11a standard. Modifications of the retransmission strategy and puncturing pattern are proposed, as well as means to combine the soft bit metrics. Contrary to classical IR which tries to have a fine granularity in the incremental retransmission process, the proposed HARQ-IR+AMC scheme balances the number of retransmissions with the need to limit the MAC overhead. The average throughput performance is evaluated under fading and interference conditions. In the presence of fading only, the MAC protocol overhead makes the throughput gain negligible. However, the HARQ-IR+AMC strategy presents an interest when interference bursts deteriorate the SNIR prediction used by the AMC. In that case, an improvement of $1.5 \mathrm{~dB}$ can be observed. Overall, this scheme improves the system robustness to interference, at the expense of only slight modifications to the standard.

\section{References}

[1] IEEE 802.11a. High Speed Physical Layer in the 5GHz band. Draft Supplement to Standard IEEE 802.11, IEEE, New York, January 1999.

[2] M. Alard and R. Lassale. Principles of Modulation and Channel Coding for Digital Broadcasting for Mobile Receivers. European Broadcasting Union Review Technical, 224:168-190, August 1987.

[3] D. Chase. Code combining. A maximum-likelihood decoding aproach for combining an arbitrary number of noisy packets. IEEE Trans. on Communications, 33:385-393, May 1985.

[4] ETSI Normalization Commitee. Broadband Radio Access Networks (BRAN); HIPERLAN Type 2; Physical (PHY) Layer. Norme ETSI, document RTS0023003-R2, European Telecommunications Standards Institute, Sophia-Antipolis, Valbonne, France, February 2001.

[5] N.C. Ericsson, A. Ahlen, S. Falahati, and A. Svensson. Hybrid type-II ARQ/AMS supported by Channel predictive Scheduling in a Multi-User Scenario. volume 4, pages 1804-1811, 2000.

[6] Sorour Falahati and Arne Svensson. Hybrid type-II ARQ schemes with adaptive modulation systems for wireless channels. volume 5, pages $2691-2695,1999$.

[7] A. J. Goldsmith and S. Chua. Adaptive coded modulation for fading channels. IEEE Trans. on Communications, 46:595-602, May 1998. 
[8] J. Haugenauer. Rate-compatible Punctured Convolutional Codes (RCPC Codes) and their applications. IEEE Trans. on Communications, 36:389-400, April 1988.

[9] Mattias Lampe, Hermann Rohling, and Wolfgand Zirwas. Misunderstandings about Link Adaptation for frequency selective fading channels. 2002.

[10] S. Lin and D. J. Costello. Error Control Coding: Fundamentals and Applications. Englewood Cliffs, NJ: Prentice-Hall, 1983.

[11] D.M. Maldelbaum. Adaptive-feedback coding scheme for hybrid ARQ systems. IEEE Trans. on Information Theory, 20:388-389, May 1974.

[12] S. Simoens and D. Bartolomé. Optimum performance of link adaptation in HIPERLAN/2 networks. In Proceedings of the IEEE Vehicular Technology Conference, Rhodes, Greece, May 2001.

[13] E. Calvanese Strinati, J. Gosteau, S. Simoens, and P. Pellati. Impact of SR-ARQ with finite buffer on TDD/TDMA Wireless LAN. In Proceedings of the IEEE Multiaccess, Mobility and Teletraffic for Wireless Communications, volume 6, Rennes, France, June 2002.

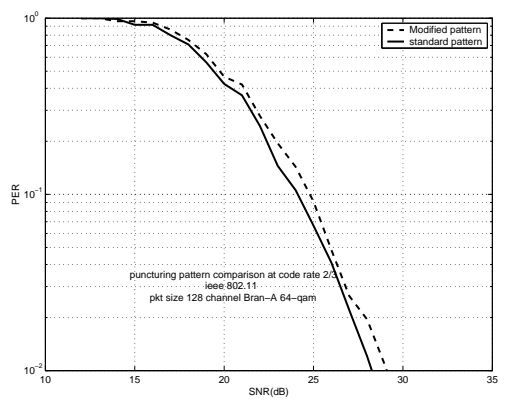

Figure 1: PER performance for standard and ratecompatible puncturing patterns

$64 \mathrm{QAM}, R=\frac{2}{3}$, BRAN-A channel, 128 byte packets

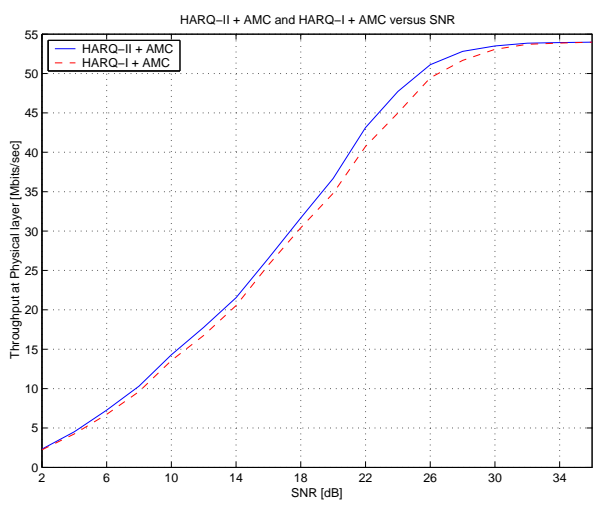

Figure 2: Impact of new HARQ-IR scheme on physical layer throughput (no interference, perfect SNIR estimation and prediction)

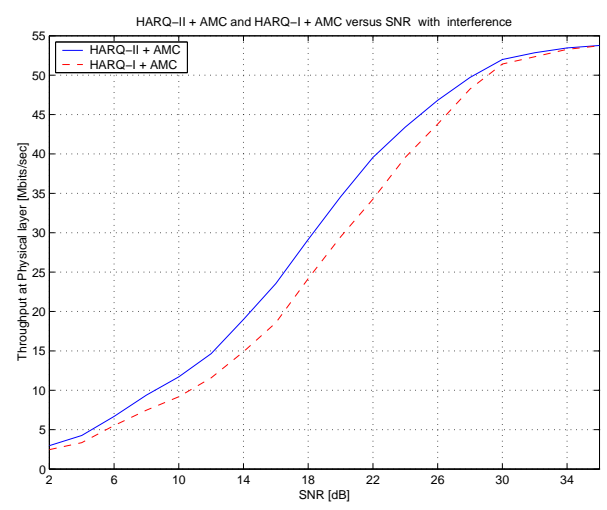

Figure 3: Impact of new HARQ-IR scheme on physical layer throughput with Interference and SNIR prediction error

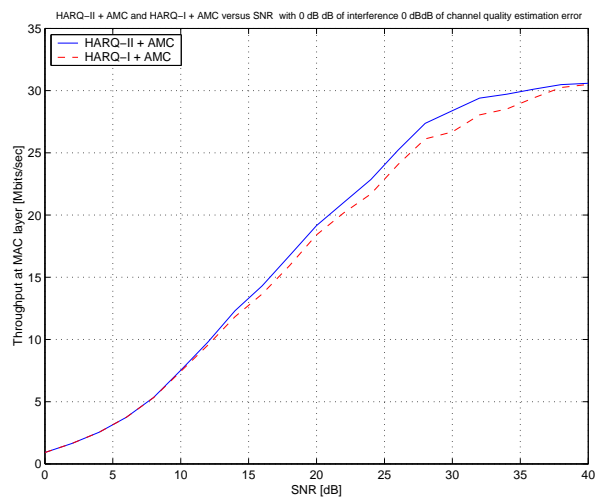

Figure 4: Impact of new HARQ-IR scheme on MAC layer throughput (no interference, perfect SNIR estimation and prediction)

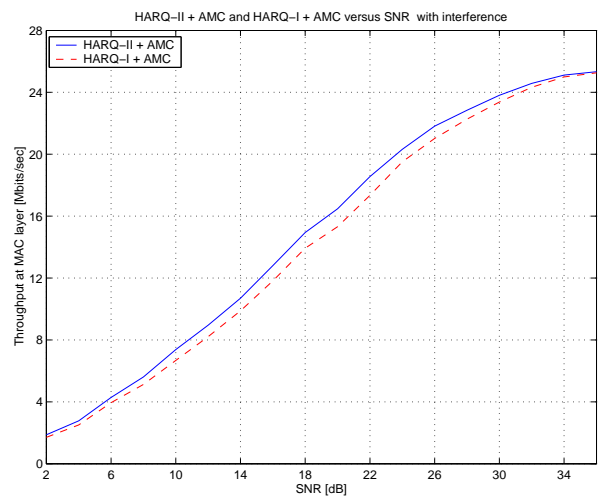

Figure 5: Impact of new HARQ-IR scheme on MAC layer throughput with Interference and SNIR prediction error 